\title{
SPACES DETERMINED BY THEIR HOMEOMORPHISM GROUPS $\left({ }^{1}\right)$
}

\author{
BY \\ E. S. THOMAS, JR.
}

1. Introduction. Let $F$ be a topological space and let $G_{F}$ denote the group of homeomorphisms of $F$ onto itself. We give $G_{F}$ the point-open topology (the topology of pointwise convergence on $F$ ) and consider it as a topological space. In general, with this topology, $G_{F}$ is not a topological group; for example, if $F$ is the unit square, inversion is not continuous.

We are concerned with finding conditions such that if $F^{\prime}$ and $F$ satisfy these conditions then an isomorphism of $G_{F^{\prime}}$ onto $G_{F}$ which is also a homeomorphism induces a homeomorphism of $F^{\prime}$ onto $F$. Some conditions are needed since there is such an isomorphism between the group of the open unit interval and that of the closed unit interval.

In [2], Wechsler showed that a sufficient condition for Hausdorff, nondiscrete spaces is $\omega$-homogeneity, i.e., for each $n$, and each pair of $n$-tuples of distinct points $\left(x_{1}, \ldots, x_{n}\right),\left(y_{1}, \ldots, y_{n}\right)$ there is a homeomorphism $h$ such that $h\left(x_{i}\right)=y_{i}$ for $i=1, \ldots, n$.

Our main result is to replace $\omega$-homogeneity by the following two conditions.

Condition A. Let $P$ and $Q$ be finite disjoint sets, let $y$ be a point of $F-P$, and let $V$ be an open set in $F$. If some member of $G_{F}$ maps $y$ into $V$ then some member of $G_{F}$ maps $y$ into $V-Q$ leaving $P$ pointwise fixed.

CoNDITION B. If $\left\{x_{\lambda}\right\}$ is a net in $F$ not converging to $x \in F$ then there is a subnet $\left\{y_{\mu}\right\}$ of $\left\{x_{\lambda}\right\}$ and a map $g \in G_{F}$ such that $g\left(y_{\mu}\right)=y_{\mu}$ for all $\mu$ and $g(x) \neq x$.

Frequently our arguments parallel those in [2] although Condition A is a good deal weaker than $\omega$-homogeneity and applies to a much wider class of spaces.

$\S 2$ consists of preliminary results. In $\S 3$ we prove the main theorems of this paper, Theorems 3.1 and 3.2. In the second of these we show that Condition B may be replaced by 1 -homogeneity. In $\$ 4$ we give examples of spaces which are not $\omega$-homogeneous but which satisfy $\mathrm{A}$. These include certain manifolds and manifoldlike spaces.

Finally, in $\$ 5$ we introduce the notion of a determining group and using this notion and our previous results we prove that no conditions whatever are needed if $F^{\prime}$ and $F$ are manifolds of dimension at least 3 .

Notation AND CONVEntions. Most of our notation can be found in [2]; for completeness a summary is given here.

Received by the editors March 24, 1966.

(1) Supported by National Science Foundation Grant GP 5935. 
All spaces are assumed to be $T_{1}$, so that complements of finite sets are open. The point-open topology for $G_{F}$ has a basis of sets of the form

$$
\left\{f \in G_{F} \mid f\left(x_{i}\right) \in U_{i}, i=1, \ldots, n\right\}
$$

where the $x_{i}$ are in $F$ and the $U_{i}$ are open in $F$. If the number of points in the basis element is irrelevant the above set will be denoted $W\left[x_{i}, U_{i}\right]$. For convenience we adopt the convention that the $x_{i}$ appearing in the basis element are distinct.

The notation $\bar{x}$ is used for a point of the $k$-fold Cartesian product $F^{k}$, no two coordinates of which are equal (what $k$ is will be clear from the context); the $i$ th coordinate of $\bar{x}$ is denoted $x_{i}$. Similarly $\bar{U}$ stands for the open set $U_{1} \times \cdots \times U_{k}$ where each $U_{i}$ is open in $F$. Thus $W[\bar{x}, \bar{U}]$ represents a typical basis element in $G_{F}$. $G_{F}$ acts on $F^{k}$ coordinatewise; i.e., if $H \subseteq G_{F}$ then $H(x)=\left\{\left(h\left(x_{1}\right), \ldots, h\left(x_{k}\right)\right) \mid h \in H\right\}$ and if $h \in H$ then $h(\bar{U})=h\left(U_{1}\right) \times \cdots \times h\left(U_{k}\right)$.

The composition of functions is denoted by juxtaposition; $g f(x)=g(f(x))$.

By "manifold" we mean a topological $n$-manifold; we make no a priori connectedness or boundary assumptions. Many of the topological concepts we use are to be found in [1].

REMARK. For the applications of the next section the following version of Condition $\mathrm{A}$ is more suitable than the simple form given above. Let $x_{1}, \ldots, x_{n}, y_{1}, \ldots$, $y_{m}$ be distinct points and let $V_{1}, \ldots, V_{m}$ be open sets such that, for $i=1, \ldots, m$, $G_{F}\left(y_{i}\right) \cap V_{i} \neq \varnothing$. Given any finite set $Q$, there is $h$ in $G_{F}$ such that $h\left(x_{i}\right)=x_{i}$ for $i=1, \ldots, n$ and $h\left(y_{i}\right) \in V_{i}-Q$ for $i=1, \ldots, m$. This statement follows from repeated applications of the original Condition $A$.

The author wishes to thank both M. T. Wechsler, who read a preliminary version of this paper and suggested the use of determining subgroups in Theorem 5.1, and the referee whose comments shortened and improved several proofs.

2. Preliminary results. Throughout this section, $x$ denotes a point of a space $F$ which satisfies Condition A.

We begin by establishing two lemmas of a technical nature. The first is used throughout the paper, the second in the proof of Theorem 2.3.

Lemma 2.1. Let $W=W[\bar{x}, \bar{U}]$ be nonvoid in $G_{F}$ and let $g$ be a member of $G_{F}$ such that $g(x), x_{1}, \ldots, x_{k}$ are distinct $\left(\bar{x}=\left(x_{1}, \ldots, x_{k}\right)\right)$. There is $f$ in $W$ such that $f(g(x))=x$.

Proof. Choose $h \in W$ and let $h^{-1}(\bar{U})=\bar{V}$. Now $W\left[g^{-1}(\bar{x}), \bar{U}\right]$ contains $h g$, hence is nonvoid, and the points $x, g^{-1}\left(x_{1}\right), \ldots, g^{-1}\left(x_{k}\right)$ are distinct, so it follows from Condition $\mathrm{A}$ that there is $f_{0}$ in $G_{F}$ such that $f_{0}(x)=x$ and $\left(f_{0} g^{-1}\right)(\bar{x}) \in \bar{U}$. Let $f=f_{0} g^{-1}$, then $f(g(x))=f_{0}(x)=x$ and $f(\bar{x})=f_{0}\left(g^{-1}(\bar{x})\right) \in \bar{U}$, i.e., $f \in W$.

LEMMA 2.2. Let $g$ be in the open set $W=W[\bar{x}, \bar{U}]$. There is a neighborhood $U$ of $x$ in $F$ such that if $y \in U \cap G_{F}(x)$ then for some $f$ in $G_{F}, f(x)=y$ and $f \in W\left[\bar{x}, g^{-1}(\bar{U})\right]$.

Proof. First suppose $x \neq x_{i}$ for all $i$. Let $U=F-\left\{x_{1}, \ldots, x_{k}\right\}$, thus $U$ is open and 
contains $x$. Suppose $y \in U \cap G_{F}(x)$, say $y=h(x)$ for some $h$ in $G_{F}$. Since $W\left[\bar{x}, g^{-1}(\bar{U})\right]$ is nonvoid (it contains the identity map of $F$ ) and since the points $x=h^{-1}(y), x_{1}, \ldots, x_{k}$ are distinct, Lemma 2.1 implies that there is $f$ such that $f\left(h^{-1}(y)\right)=y$ and $f \in W\left[\bar{x}, g^{-1}(\bar{U})\right]$. Since $h^{-1}(y)=x$, we are done in this case.

Now suppose $x=$ some $x_{i}$, say $x=x_{1}$. Let $U=F-\left\{x_{2}, \ldots, x_{k}\right\} \cap g^{-1}\left(U_{1}\right)$. Again $U$ is a neighborhood of $x$. If $y \in U \cap G_{F}(x)$ and $y \neq x$ then, as above, Lemma 2.1 applies to give the desired $f$. If $y=x$ then we take $f$ to be the identity map.

The next result gives a relationship between certain quotient spaces of $G_{F}$ and subsets of $F$. It is the analogue of Theorem 2.6 of [2]. We use the following notation. If $H$ is a subgroup of $G_{F}$ then $G_{F} / H$ is the collection of left cosets with the quotient topology. For each $x \in F$ the subgroup of maps leaving $x$ fixed is denoted $G_{x}$, and $\theta_{x}$ is the map of $G_{F} / G_{x}$ into $F$ given by: $\theta_{x}\left(g G_{x}\right)=g(x)$.

THEOREM 2.3. $\theta_{x}$ is a homeomorphism of $G_{F} / G_{x}$ onto $G_{F}(x)$.

Proof. It is easy to see that $\theta_{x}$ is well defined, one-to-one, and onto.

Let $\nu$ denote the quotient map of $G_{F}$ onto $G_{F} / G_{x}$. The map $\theta_{x}$ is continuous if the composition $\theta_{x} \nu$ is [1, Theorem 9, p. 95] and a simple argument shows that if $\theta_{x} \nu$ is open then so is $\theta_{x}$.

Thus, continuity of $\theta_{x}$ follows immediately from the fact that $G_{F}$ has the pointopen topology. To see that $\theta_{x}$ is open, let $W=W[\bar{x}, \bar{U}]$ be a basis element for the point-open topology and let $V=\theta_{x} \nu(W)=\{g(x) \mid g \in W\}$. We must show $V$ is open in the relative topology for $G_{F}(x)$.

Suppose $y \in V$, say $y=g(x)$ for some $g$ in $W$. Choose an open set $U$ in $F$ satisfying the requirements of Lemma 2.2. Then $\tilde{U}=U \cap G_{F}(x)$ is a neighborhood of $x$ in $G_{F}(x)$ and $g(\tilde{U})$ is a neighborhood of $y$ in $G_{F}(x)$. We show that $g(\tilde{U}) \subseteq V$. Let $z$ be in $g(\tilde{U})$, say $z=g(w)$ for some $w \in \tilde{U}$. Choose $f$ such that $f(x)=w$ and $f \in W\left[\bar{x}, g^{-1}(\bar{U})\right]$ and let $h=g f$. Then $h \in W[\bar{x}, \bar{U}]=W$ and $h(x)=g f(x)=g(w)=z$. Since $z$ was arbitrary in $g(\tilde{U})$ we have shown that $g(\tilde{U}) \subseteq V$. Since $y$ was arbitrary in $V$ this shows $V$ is open in $G_{F}(x)$ and completes the proof of the theorem.

The final result of this section is crucial in the proof of the main theorem. Points $\bar{x}$ and $\bar{y}$ of $F^{k}$ are independent provided no component of $\bar{x}$ is a component of $\bar{y}$.

LEMMA 2.4. Let $H$ be a subgroup of $G_{F}$ and suppose $W[\bar{x}, \bar{U}]$ is a nonvoid open set missing $H$ where $\bar{x} \in F^{k}, k \geqq 1$. Suppose there is $\bar{y}$ in $F^{k}$ such that $\bar{y}$ is in $H(\bar{x})$ and $\bar{x}$ and $\bar{y}$ are independent. Then $\bar{x}$ lies in infinitely many distinct sets of the form $g H(\bar{x})$.

Proof. $\bar{x}$ is certainly in $H(\bar{x})$. Suppose we have $n$ distinct sets $g_{1} H(\bar{x}), g_{2} H(\bar{x}), \ldots$, $g_{n} H(\bar{x})(n \geqq 1)$ containing $\bar{x}$; we show there is $g_{n+1} H(\bar{x})$, distinct from the previous sets, containing $\bar{x}$.

Case 1. Suppose $H(\bar{x})$ contains $n+1$ independent elements, say $\bar{x}, \bar{x}_{1}, \ldots, \bar{x}_{n}$. For each $i=1, \ldots, n$ write $\bar{x}_{i}=\left(y_{1}^{i}, \ldots, y_{k}^{i}\right)$. For fixed $i$ and any $j(1 \leqq j \leqq k)$, $G_{F}\left(y_{j}^{i}\right)=G_{F}\left(x_{j}\right)$ meets $U_{j}$ and hence meets $g_{i}\left(U_{j}\right)$. By Condition A there is a function 
$g_{n+1}$ such that $g_{n+1}\left(x_{i}\right)=x_{i}$ for $i=1, \ldots, k$ and $g_{n+1}\left(y_{j}^{i}\right) \in g_{i}\left(U_{j}\right)$ for $i=1, \ldots, n$ and $j=1, \ldots, k$. Thus $g_{n+1}(\bar{x})=\bar{x}$ and for $i=1, \ldots, n, g_{n+1}\left(\bar{x}_{i}\right) \in g_{i}(\bar{U})$. The first condition on $g_{n+1}$ implies that $\bar{x} \in g_{n+1} H(\bar{x})$. The second condition implies that $g_{n+1} H(\bar{x})$ is distinct from $g_{i} H(\bar{x})$ for $1 \leqq i \leqq n$, because $g_{n+1}\left(\bar{x}_{i}\right)$ meets $g_{i}(\bar{U})$ while $g_{i}\left(\bar{x}_{i}\right)$ does not ( $H$ misses $\left.W[\bar{x}, \bar{U}]\right)$.

Case 2. Suppose $H(\bar{x})$ contains at most $n$ independent elements. The same is true of the sets $g_{i} H(\bar{x}), i=1, \ldots, n$. It follows that there is a finite set $Q \subset F$ such that if $\bar{w}=\left(w_{1}, \ldots, w_{k}\right)$ is in $F^{k}$ and no $w_{i}$ is in $Q$ then $\bar{w}$ is in no $g_{i} H(\bar{x})$. We have independent points $\bar{x}$ and $\bar{y}$ in $H(\bar{x})$; apply Condition A to get a map $g_{n+1}$ such that $g_{n+1}(\bar{x})=\bar{x}$ and $g_{n+1}\left(y_{i}\right) \in F-Q$ for $i=1, \ldots, k$ (where $\bar{y}=\left(y_{1}, \ldots, y_{k}\right)$ ). Since $g_{n+1}(\bar{y})$ is no $g_{i} H(\bar{x})$, for $1 \leqq i \leqq n, g_{n+1} H(\bar{x})$ is distinct from $g_{1} H(\bar{x}), \ldots, g_{n} H(\bar{x})$ and we are done.

\section{The main theorem.}

THEOREM 3.1. Let $F^{\prime}$ and $F$ be spaces satisfying Condition $\mathrm{A}$ and let $\Phi$ be an isomorphism of $G_{F^{\prime}}$ onto $G_{F}$ which is also a homeomorphism. For each $x$ in $F^{\prime}$, $\Phi\left(G_{x}\right)$ is the subgroup $G_{y}$ of a point $y$ of $F$. The induced function $x \rightarrow y$ from $F^{\prime}$ into $F$ is one-to-one and onto.

Proof. Fix $x \in F^{\prime}$ and let $H=\Phi\left(G_{x}\right)$; thus $H$ is a proper closed subgroup of $G_{F}$ and, for some $n$, there exist $\bar{x} \in F^{n}$ and $\bar{U} \subset F^{n}$ such that the nonvoid basic open set $W=W[\bar{x}, \bar{U}]$ in $G_{F}$ misses $H$. We assume $n$ is the smallest integer with this property.

We first show $n=1$ and $H(\bar{x})$ is a single point of $F^{1}=F$. Suppose not; then $H(\bar{x})$ contains two independent points $\bar{x}$ and $\bar{y}$. (This is trivial if $n=1$ while, if $n>1$, it follows from Lemma 3.14 of [2].) By our Lemma 2.4 there exist infinitely many distinct sets $\left\{g_{i} H(\bar{x}) \mid i=1,2, \ldots\right\}$ each containing $\bar{x}$. In particular, the cosets $\left\{g_{i} H \mid i=1,2, \ldots\right\}$ are distinct.

Let $V=\Phi^{-1}(W)$; since $V$ is open in $G_{F^{\prime}}$ it contains a nonvoid basic open set, $W\left[y_{i}, U_{i}\right], i=1, \ldots, m$. Let $h_{i}=\Phi^{-1}\left(g_{i}\right)$ for $i=1,2, \ldots$ Since the sets

$$
\left\{h_{i} G_{x} \mid i=1,2, \ldots\right\}
$$

are distinct, we may choose $k$ so that $h_{k}(x), y_{1}, \ldots, y_{m}$ are distinct. By Lemma 2.1 there is $g \in G_{F^{\prime}}$ such that $g \in W\left[y_{i}, U_{i}\right] \subset V$ and $g h_{k}(x)=x$, i.e., $g h_{k} G_{x}=G_{x}$. Thus $f=\Phi(g)$ is a map in $G_{F}$ such that $f \in \Phi(V)=W$ and $f g_{k} H=H$. Since $\bar{x} \in g_{k} H(\bar{x})$, we have from the last condition that $f(\bar{x}) \in H(\bar{x})$. But $H(\bar{x}) \cap \bar{U}=\varnothing$ and $W=W[\bar{x}, \bar{U}]$; so we have a contradiction.

To summarize, we have shown that $H(\bar{x})$ is a single point of $F$, say $H(\bar{x})=\{y\}$. It follows that $H \subseteq G_{y}$ and we next show that $H=G_{y}$.

Suppose there is $g \in G_{y}-H$, then putting $f=\Phi^{-1}(g)$ we have $f \in G_{F^{\prime}}-G_{x}$. We show that $G_{x} f G_{x}$ is dense in $G_{F^{\prime}}$. Let $W=W\left[x_{i}, U_{i}\right], i=1, \ldots, n$, be nonvoid and open in $G_{F^{\prime}}$. Since $f(x) \neq x$ there is, by Condition A, a map $h_{1}$ such that $h_{1}(x)=x$ and $h_{1}\left(x_{i}\right) \neq f^{-1}(x)$ for $i=1, \ldots, n$; i.e., $h_{1} \in G_{x}$ and $f h_{1}\left(x_{i}\right) \neq x$ for $i=1, \ldots, n$. Now again by Condition A, there is $h_{2}$ such that $h_{2}(x)=x$ and $h_{2} f h_{1}\left(x_{i}\right) \in U_{i}, i=1, \ldots, n$. 
Thus $h_{2} f h_{1} \in G_{x} f G_{x} \cap W$ and $G_{x} f G_{x}$ is dense. But then $\Phi\left(G_{x} f G_{x}\right)$ is dense in $G_{F}$ which is absurd since $\Phi\left(G_{x} f G_{x}\right) \subseteq H G_{y} H=G_{y}$ which is a proper closed subgroup of $G_{F}$.

Thus we have: $\Phi\left(G_{x}\right)=G_{y}$. Obviously a dual result holds for $\Phi^{-1}$ so that the induced correspondence $x \rightarrow y$ from $F^{\prime}$ into $F$ is one-to-one and onto.

THEOREM 3.2. Let $F^{\prime}, F$, and $\Phi$ be as in Theorem 3.1, and let $\phi$ be the map of $F^{\prime}$ onto $F$ given by: $\phi(x)=y$ where $\Phi\left(G_{x}\right)=G_{y}$. Then $\phi$ is a homeomorphism if (i) both spaces satisfy Condition $\mathrm{B}$, or, (ii) both spaces are 1-homogeneous.

REMARK. If both spaces are assumed to satisfy the first axiom of countability then Condition B can be replaced by the corresponding statement for sequences.

Proof. Suppose Condition B holds in $F$ and let $\left\{x_{\lambda}\right\}$ be a net in $F^{\prime}$ converging to $x$. If $\left\{\phi\left(x_{\lambda}\right)\right\}$ does not converge to $\phi(x)$ in $F$ then there is a subnet $\left\{y_{\mu}\right\}$ of $\left\{\phi\left(x_{\lambda}\right)\right\}$ and a function $g$ in $\bigcap_{\mu} G_{y_{\mu}}-G_{\phi(x)}$. Put $z_{\mu}=\phi^{-1}\left(y_{\mu}\right)$, then $\left\{z_{\mu}\right\}$ is a subnet of $\left\{x_{\lambda}\right\}$ and $\Phi^{-1}(g)$ is in $\bigcap_{\mu} G_{z_{\mu}}-G_{x}$. Since $\left\{z_{\mu}\right\}$ converges to $x$ this is impossible. Thus $\phi$ is continuous. Dually, if $F^{\prime}$ satisfies Condition B then $\phi^{-1}$ is continuous.

For the second part of the proof, assume both spaces are 1-homogeneous. Fix a point $x$ of $F^{\prime}$; then we have:

$$
F^{\prime}=G_{F^{\prime}}(x) \approx G_{F^{\prime}} / G_{x} \approx G_{F} / G_{\phi(x)} \approx G_{F}(\phi(x))=F,
$$

where $\approx$ denotes a homeomorphism. The first and last equality signs follow from 1-homogeneity, the first and last homeomorphisms from Theorem 2.3, and the middle homeomorphism is induced by $\Phi$. The composition of these maps yields a homeomorphism $\psi$ and we assert that $\psi=\phi$. It is enough to prove that for each $g$ in $G_{F^{\prime}}, \psi(g(x))=\phi(g(x))$. One easily verifies that $\psi(g(x))=\Phi(g)(\phi(x))$. This last expression is $\phi(g(x))$ if and only if $\Phi\left(G_{g(x)}\right)=G_{\Phi(g)(\phi(x))}$. But

$$
\Phi\left(G_{g(x)}\right)=\Phi\left(g G_{x} g^{-1}\right)=\Phi(g) G_{\phi(x)} \Phi(g)^{-1}=G_{\Phi(g)(\phi(x))}, \quad \text { Q.E.D. }
$$

4. Condition A. Most of this section is devoted to exhibiting spaces to which Theorem 3.1 applies, i.e., spaces in which Condition A holds. At the end we give a condition which is necessary in order that a space satisfy Condition A.

We remark that Theorem 2.3 applies to all spaces mentioned below. In all these spaces Condition B holds although the second part of the theorem applies directly in the $\omega$-homogeneous case.

To begin with, any $\omega$-homogeneous space satisfies Condition $\mathrm{A}$; this includes all connected manifolds without boundary of dimension at least 2, the Hilbert cube, and some totally disconnected spaces such as the Cantor set or the set of points with rational coordinates in $E^{n}$.

More generally let $M$ be a connected manifold whose boundary $\partial M$ has dimension at least 2 and suppose no two components of $\partial M$ are homeomorphic, then $M$ satisfies Condition A. 
Question. Let $M$ be a connected manifold of dimension at least 3 and let $K$ be a subset of a component of $\partial M$. If $K$ satisfies Condition A then does $\stackrel{M}{ } \cup K$ satisfy Condition A?

Spaces satisfying the condition can be manufactured by patching together manifolds of different dimensions. As an example, let $F=\widetilde{B}^{4} \cup T$ where $\widetilde{B}^{4}$ is the standard 4-ball in $E^{4}$ with the north pole removed and $T$ is a 3-cell in $E^{4}$ such that $T \cap B^{4}=\partial T$ is the 2 -sphere $\left\{\left(x_{1}, \ldots, x_{4}\right) \in \partial B^{4} \mid x_{4}=0\right\}$.

It should be pointed out that a space need not be connected or totally disconnected in order that it satisfy Condition A. Let $F$ be a space each component of which is open in $F$ and satisfies Condition A; then if no two components of $F$ are homeomorphic, $F$ satisfies A.

We end this section by giving a necessary condition that a space satisfy Condition A. This is a take-off on the fact that a circle is not 4-homogeneous. Let us say that a subset $S$ of a space $F$ contains a free arc if it contains an arc $A$ with endpoints $a$ and $b$ such that, with the relativized topology, $A-\{a, b\}$ is open in $S$. Our condition can be stated as follows: In order that $F$ satisfy Condition A it is necessary that, for each $x$ in $F, G_{F}(x)$ contains no free arc.

We sketch a proof of this fact. Suppose $\gamma$ is a homeomorphism of $I=[0,1]$ into $F$ such that $\gamma(I) \subseteq G_{F}(x)$ for some $x$ and $\gamma((0,1))$ is open relative to $G_{F}(x)$. Let $y=\gamma(0), x_{1}=\gamma(1 / 4), x_{2}=\gamma(1 / 2), x_{3}=\gamma(3 / 4)$ and let $U$ be open in $F$ such that $U \cap G_{F}(x)=\gamma((1 / 4,1 / 2))$. Since $G_{F}(x)=G_{F}(y)$ we have $G_{F}(y) \cap U \neq \varnothing$. One then shows by an order argument that no member of $G_{F}$ (indeed, no self-homeomorphism of $\left.G_{F}(x)\right)$ leaves $x_{1}, x_{2}$, and $x_{3}$ fixed and takes $y$ into $U$; thus Condition A fails.

5. A better theorem for manifolds. In the preceding sections we have not used the hypothesis that $G_{F}$ consists of all self-homeomorphisms of $F$. This fact is important; we shall show that for manifolds a sharpening of the previous results is obtained by using fewer homeomorphisms.

A subgroup $H$ of $G_{F}$ is a determining group if Conditions $\mathrm{A}$ and B hold with $G_{F}$ replaced by $H$. Clearly what is proved in $\$ \S 2$ and 3 is that if $H^{\prime}$ and $H$ are determining groups for spaces $F^{\prime}$ and $F$ then a map $\Phi$ of $H^{\prime}$ onto $H$ which is an isomorphism and a homeomorphism induces a homeomorphism of $F^{\prime}$ onto $F$.

Using this notion we circumvent Conditions A and B as hypotheses to obtain the following very general theorem.

THEOREM 5.1. Let $M$ and $N$ be manifolds of dimension at least 3 (with or without boundary, connected or disconnected) and suppose $\Phi$ is an isomorphism and homeomorphism of $G_{M}$ onto $G_{N}$. Then $\Phi$ induces a homeomorphism of $M$ onto $N$.

Proof. Let $C_{M}$ denote the component of the space $G_{M}$ containing the identity map $e_{M}$ of $M$ and define $C_{N}$ similarly. Then $\Phi\left(C_{M}\right)=C_{N}$. We shall show below that $C_{M}$ and $C_{N}$ are determining subgroups for $M$ and $N$, hence, by the above remarks, there is an induced homeomorphism as asserted. 
Let $H_{M}$ denote the subgroup of $G_{M}$ consisting of all homeomorphisms leaving each component of $M$ and each component of $\partial M$ fixed. (If $M$ or $\partial M$ is connected delete the corresponding restriction.) We prove that $H_{M}=C_{M}$. It is easy to see that $H_{M}$ is a closed subgroup of $G_{M}$ containing $e_{M}$. If $g \in G_{M}-H_{M}$ then there exist distinct components $K$ and $K^{\prime}$ of either $M$ or $\partial M$ such that $g(K)=K^{\prime}$. Pick $x \in K$ and let $U$ be an open set containing $K^{\prime}$ and missing every component of $M$ or $\partial M$ which misses $K^{\prime}$. Then $W[x, U]$ is an open and closed set in $G_{M}$ containing $g$ and missing $H_{M}$. This implies that $C_{M} \subseteq H_{M}$. To complete the proof it suffices to show $H_{M}$ is connected.

Let $W=W\left[x_{i}, U_{i}\right]$ be open in $G_{M}$ and suppose $W \cap H_{M}$ is nonvoid. Thus, each $U_{i}$ meets the component of $M$ containing $x_{i}$ and, if $x_{i} \in \partial M$, then $U_{i}$ meets the component of $\partial M$ containing $x_{i}$. From these facts and the fact that $M$ has dimension at least 3 we can obtain an isotopy $\left\{g_{t} \mid 0 \leqq t \leqq 1\right\}$ with the following properties: $g_{0}=e_{M}, g_{t} \in H_{M}$ for all $t$ and $g_{1}\left(x_{i}\right) \in U_{i}$ for each $i$. This isotopy is a connected subset of $G_{M}$, lying in $H_{M}$, joining $e_{M}$ to $W$. It follows that $H_{M}$ is connected, thus $H_{M}=C_{M}$.

- Clearly the dimension requirements insure that $H_{M}$ is a determining group. A dual result holds for $N$ and the proof is complete.

\section{BIBLIOGRAPHY}

1. J. L. Kelly, General topology, Van Nostrand, New York, 1955.

2. M. T. Wechsler, Homeomorphism groups of certain topological spaces, Ann. of Math. 62 (1955), 360-373.

UNIVERSITY OF MICHIGAN, ANn Arbor, Michigan 